\title{
La Autonomía en la Educación Superior: Reflexiones desde los Actores en el Contexto del Financiamiento por Desempeño en Chile
}

\author{
Carmen A. Araneda-Guirriman ${ }^{(1)}$, Joaquín Gairín-Sallán ${ }^{(2)}$ y Liliana M. Pedraja-Rejas ${ }^{(1)}$ \\ (1) Escuela Universitaria de Ingeniería Industrial, Informática y Sistemas, Universidad de Tarapacá. Casilla \\ 7-D Arica, Chile. (e-mail: caraneda@uta.cl; Ipedraja@uta.cl) \\ (2) Facultad de Ciencias de la Educación, Universidad Autónoma de Barcelona, Campus de Bellaterra, \\ edificio G-5, 08193 Bellaterra-Cerdanyola del Vallés, España. (e-mail:joaquin.gairin@uab.cat)
}

Recibido Dic. 22, 2017; Aceptado Mar. 14, 2018; Versión final May. 24, 2018, Publicado Ago. 2018

\begin{abstract}
Resumen
Esta investigación explora la percepción que tienen los miembros de la comunidad académica respecto a la autonomía de las universidades en el contexto del financiamiento por desempeño en Chile. Para este fin, se realizaron entrevistas semiestructuradas a veinte miembros de la comunidad académica de dos universidades estatales chilenas. El método utilizado para la realización de este estudio fue la Teoría Fundamentada, donde los procesos de muestreo, recolección y análisis se encuentran estrechamente vinculados. La información recolectada fue transcrita y procesada en el software NVivo 11. Aquí se procedió primero a la construcción del descubrimiento de conceptos, a través del método de comparación constante, generando posteriormente la construcción de categorías en nodos libres. Los resultados indican que la autonomía en el contexto de financiamiento por desempeño se encuentra estrechamente vinculada al concepto de rendición de cuentas, y que esta ocurre al interior de un marco regulatorio definido por el Estado.
\end{abstract}

\section{Autonomy in Higher Education: Reflections from the Actors in the Context of Performance-based Funding in Chile}

\begin{abstract}
This research explores the perception that members of the academic community have regarding the autonomy of universities in the context of performance-based funding in Chile. For this purpose, semistructured interviews were carried out with twenty members of the academic community of two Chilean state universities. The method used to carry out this study was the Grounded Theory, where the processes of sampling, collection, and analysis are closely linked. The information collected was transcribed and processed using the NVivo 11 software. Here the construction was first performed to the discovery of concepts, through the constant comparison method, subsequently generating the categories in free nodes. The results indicate that autonomy in the context of financing by performance is closely linked to the concept of accountability and that this occurs within a regulatory framework defined by the State.
\end{abstract}

Keywords: higher education; funding; autonomy; grounded theory 


\section{INTRODUCCIÓN}

Los responsables de definir las políticas públicas han estado preocupados durante décadas por incrementar la eficiencia y la eficacia en las instituciones de educación terciaria, como sucede en Estados Unidos (Dougherty et al., 2016). Particularmente y en un contexto de presupuestos públicos restringidos, como ocurre en Europa, el financiamiento basado en el desempeño de las universidades es percibido frecuentemente como una herramienta útil por los delimitadores de políticas públicas, al considerar que conecta el financiamiento con indicadores medibles que incrementan la transparencia del gasto e incentiva y resguarda el logro de objetivos específicos de la política pública (Estermann et al., 2013). En este sentido, las preocupaciones respecto al desempeño y los costos de eficiencia han ocupado el centro de las discusiones sobre el financiamiento y el monitoreo a las universidades públicas. En Estados Unidos, las preocupaciones mencionadas se manifiestan así en las políticas de financiamiento por desempeño o en las políticas que buscan asociar de manera directa las asignaciones estatales con los resultados institucionales logrados por los estudiantes (Rutherford y Rabovsky, 2014). De esta manera, se vincula el financiamiento con los resultados de la institución, promoviendo la reflexión alrededor de los desafíos que implica conducir a las instituciones universitarias bajo estos nuevos términos de relación y financiamiento.

Las actuales reformas universitarias y de relación entre el Estado y la universidad, en el sentido mencionado, proporcionan relevancia al concepto de autonomía (Enders et al., 2013). La autonomía de las instituciones de educación superior se ve ligada al incremento de la rendición de cuentas, particularmente con respecto al uso de los fondos públicos, la posibilidad de obtener beneficios, un mayor control sobre los recursos y la libertad de elegir inversiones estratégicas (Pucciarelli y Kaplan, 2016). Chile, como país, no se encuentra exento de la realidad mencionada y se enfoca a aplicar mecanismos de financiamiento por desempeño regidos bajo los elementos de la Nueva Gestión Pública, con los nuevos retos que conlleva para el sistema de educación chileno y, entre ellos, la relevancia de las relaciones que se establecen entre las universidades y el Estado en esta lógica de financiamiento. Ciertamente, al primar aspectos como el financiamiento público, la rendición de cuentas y la definición de nuevas formas de interacción, se hace necesario reflexionar respecto a la autonomía que detentan dichas instituciones, sobre todo las de naturaleza pública donde conviven interacciones entre las que se identifican control y autonomía como un nuevo tipo de relación vinculada a los criterios de transparencia y eficiencia.

Por consiguiente, el objetivo de este trabajo, que se enmarca al interior del Programa de Doctorado en Educación de la Universidad Autónoma de Barcelona, es presentar una aproximación respecto a las percepciones que tienen los miembros de las comunidades académicas sobre el efecto que tiene la implementación de convenios de desempeño en la autonomía de las universidades que participan de este mecanismo de financiamiento. Contribuyendo de esta forma a la discusión y al acervo de conocimientos respecto a cómo influyen los instrumentos de financiamiento de la educación superior, como parte de una política pública, al interior de las universidades. Lo anterior, más específicamente, desde una perspectiva que se aproxima desde la percepción de la autonomía que tienen los integrantes de las comunidades académicas de las universidades, en un contexto de financiamiento por desempeño y rendición de cuentas en el contexto chileno, desde la perspectiva de los propios actores que participaron de este proceso.

\section{AUTONOMÍA Y FINANCIAMIENTO}

Una reflexión crítica respecto a la autonomía en el interior de las universidades estatales chilenas en el contexto del financiamiento por desempeño parece así pertinente y necesaria. La presente aportación incluye, al respecto, reflexiones sobre la temática y datos de entrevistas realizadas a miembros de la comunidad académica de dos universidades estatales chilenas. La información referenciada fue obtenida en el marco de una investigación realizada para determinar el impacto percibido de los convenios de desempeños en las universidades chilenas.

\section{Financiamiento por desempeño en la educación superior}

La financiación de la educación superior ha sido un tema recurrente en las dos últimas décadas, ligada a la masificación de la educación superior (proceso aún inacabado), el incremento de la heterogeneidad de las necesidades de los estudiantes y la respuesta de sectores de la educación más diferenciados y con un número cada vez mayor de programas y de nuevas instituciones educativas (Nagy et al., 2014). Cabe sumar, además, el cambio en la relación de las universidades con el Estado y un incremento de las transformaciones en los modos, las prácticas y las estrategias organizativas (Christopherson et al., 2014).

Los sistemas de financiamiento se han movido, en términos generales y durante las últimas cuatro décadas, desde un sistema netamente público a un sistema de financiamiento mixto, es decir, público y privado, con un mercado basado en préstamos de matrículas y donde los estudiantes graduados y las propias 
instituciones de educación superior son los responsables de una significativa proporción de los costos totales, como sucede en el caso de Reino Unido (Marginson, 2017). Dentro de los cambios experimentados en esta esfera, se encuentra la introducción del factor de desempeño de las instituciones de educación superior como elemento de ponderación para la entrega del financiamiento público.

En un contexto de mayor competencia por los recursos públicos, las preocupaciones sobre el desempeño y la rentabilidad han tomado un lugar central en las discusiones sobre el financiamiento y la supervisión de las universidades estatales. Un claro ejemplo de la manifestación de esta preocupación se encuentra en las políticas de financiamiento por desempeño, o políticas que buscan vincular las asignaciones del Estado directamente con los resultados institucionales generados por los estudiantes (Rutherford y Rabovsky, 2014). De alguna manera, las autoridades educativas están deseosas de obtener un mayor retorno del dinero invertido. Y este fenómeno es más acusado en algunos países; así y desde el 2008, la situación económica de muchos países europeos se ha deteriorado de manera considerable y las autoridades esperan, a menudo, más resultados por menos dinero (Estermann et al., 2013).

El financiamiento basado en el desempeño es entendido como un mecanismo de financiamiento de las instituciones públicas basado en resultados tales como la retención, obtención de título o la colocación laboral y no basado exclusivamente en insumos como la matrícula (Dougherty et al., 2013). Los indicadores claves de desempeño son utilizados, en este sentido, para medir el rendimiento de la organización en relación con los objetivos estratégicos y operacional, buscando medidas cuantificables que reflejen factores críticos de éxito en la universidad y que, por tanto, juegan un papel importante en el momento de evaluar el financiamiento basado en el desempeño en la educación superior (Kairuz et al., 2016). Los convenios de desempeño son considerados contratos entre el gobierno y una institución específica de educación superior, donde se establecen las metas específicas que las instituciones se proponen alcanzar en un período determinado y que constituye el desempeño u obligación a cumplir (de Boer y Jongbloed, 2014). Los contratos basados en resultados, es decir, que compensan los resultados obtenidos, son más racionales, sobre todo cuando el monitoreo es demasiado poco práctico o costoso. Por esta razón, y teniendo en cuenta la Teoría de Agencia, es que este tipo de contratos reducen el conflicto de objetivos e incentivan al agente a buscar lograr los resultados que son compatibles con los objetivos del principal (Kivistö y Zalyevska, 2015).

En el caso de Chile, existe lo que se denomina Convenio de Desempeño, que consiste en un contrato entre la universidad y el Estado mediante el que se establece un compromiso sobre los resultados notables a conseguir, que conlleven a un mejoramiento significativo de la institución y que justifique el traspaso de los recursos, buscando el incremento de la efectividad y eficiencia del gasto público, alinear los objetivos de la institución con los relevantes para el contexto nacional y fomentar la rendición de cuentas junto con el mejoramiento del rendimiento institucional (Reich et al., 2011). Este mecanismo de financiamiento se enmarca en la Nueva Gestión Pública, proceso que viene siendo implementado en Chile desde el año 1998, donde se estableció la utilización de instrumentos de evaluación de desempeño asociados a incentivos económicos (Morales, 2014). En efecto, uno de los puntos que consideró la Nueva Gestión Pública en Chile, fue la presión por resultados, siendo uno de los instrumentos preferidos la utilización de incentivos para lograr resultados (Pliscoff-Varas, 2017).

Precisamente, un convenio de desempeño en Chile y como elemento de la política pública, se convierte en un instrumento de financiamiento estatal para las instituciones de educación superior que buscan cambios relevantes. Las instituciones de educación superior que reciben los recursos públicos deben estar dispuestas a asumir, por este motivo, el compromiso de lograr desempeños notables, académicos e institucionales, negociados y sobre los cuales debe efectuarse la rendición de cuentas (Yutronic et al., 2012). El financiamiento por desempeño en materia de política de financiamiento por desempeño en Chile se ha llevado a cabo también por medio del fondo "Convenio Marco Universidades Estatales", que tiene por finalidad la celebración de convenios entre el Ministerio de Educación y las universidades estatales para el desarrollo de la institución y mediante los convenios de desempeño.

Por consiguiente, el modelo de financiamiento por desempeño constituye, además, una relación contractual más bien jerárquica, donde cada unidad organizacional involucrada en el proceso debe rendir cuenta de su desempeño y, como resultado, la rendición de cuentas y la eficiencia deben incrementarse (Barnes et al., 2014). Por rendición de cuentas, se entiende aquí y de acuerdo con la definición aportada por Trow (1996) al proceso obligatorio de reportar a otros, de justificar, explicar, responder preguntas respecto a cómo los recursos han sido utilizados y cuál es su efecto, por ende, los aspectos centrales de este proceso son: quien debe ser responsable, para qué, a quienes, a través de qué medios, con qué consecuencias. El financiamiento por desempeño es clave, en este sentido, para el mecanismo de rendición de cuentas (Letizia, 2016). 


\section{Autonomía de las instituciones de educación superior}

Las formas como se regula y funciona el financiamiento de las instituciones de educación superior quedan muy condicionadas con los grados de autonomía que se permiten. Pensar en convenios de desempeño con rendición de cuentas sólo tiene sentido en el marco de una cierta autonomía institucional. En este sentido y en un escenario de democracia liberal, la autonomía de las instituciones universitarias desempeña un papel clave, considerando que las universidades solo pueden ser pensadas como instituciones autónomas. No hablamos de ausencia de regulación ni de normas de control sino de ciertos márgenes de maniobra institucional, puesto que bajo ciertos contextos los mecanismos de control son requeridos para el mejoramiento de la calidad (Correa, 2017). Este planteamiento no es trivial y resulta sustancial, si consideramos que las universidades son instituciones sociales distintivas que merecen un trato especial en cuanto a su libertad académica y autonomía procedimental, con las que el Estado establece una asociación respecto a las políticas sustantivas, que debieran ser expresadas mediante un mecanismo adecuado (Berdahl, 1990).

Más concretamente, la Declaración de Graz (EUA, 2003), que es el mayor documento de política resultante y producto de la segunda convención de Instituciones de Educación Superior Europeas, establece que los gobiernos deben otorgar a las instituciones una cierta responsabilidad pública y fortalecer su autonomía esencial otorgándoles entornos estables tanto a nivel jurídico como de financiamiento. A su vez, establece que las universidades aceptan rendir cuentas y asumirán el deber de implementar las reformas en estrecha cooperación con los estudiantes y los grupos de interés, mejorando de esta forma la capacidad de dirección estratégica. En Chile, la autonomía de las universidades se encuentra normada por medio el Decreto con Fuerza de Ley $\mathrm{N}^{\circ} 2$, específicamente en su Artículo 104, donde se entiende por autonomía el derecho de cada establecimiento de educación superior a regirse por sí mismo, de conformidad con lo establecido en sus estatutos en todo lo concerniente al cumplimiento de sus finalidades y comprende la autonomía académica, económica y administrativa.

Se puede hablar así de un periodo crítico para la educación superior, donde se plantea lograr un reequilibrio de las relaciones de poder, autoridad y responsabilidad en la gobernanza de las instituciones de educación terciaria, situación que ocurre tanto a nivel interno como externo (Maassen, 2014). Lo anterior resulta relevante, al considerar que la relación entre universidad y Ministerio de Educación, en el contexto de la implementación de los convenios de desempeño, puede ser explicada desde la perspectiva de la Teoría de Agencia, que grafica la relación contractual entre dos o más partes, en la cual una de las partes es designada como el principal (Ministerio de Educación, en este caso) involucra a otra parte, designado como agente (universidad, en este contexto) para realizar alguna acción en nombre del principal, implementar el convenio de desempeño, donde en forma de retribución por el esfuerzo, el agente recibe un pago de parte del principal (recursos asignados para la realización de las acciones) (Kivistö y Zalyevska, 2015).

El cambio necesario en la relación de las universidades con el Estado se puede considerar como parte de la crisis del sistema universitario y se relaciona con un incremento de las presiones corporativas y transformaciones en las formas, prácticas y estrategias organizativas (Christopherson et al., 2014). Se pone de manifiesto la importancia del reconocimiento de la autonomía de las instituciones de educación superior como parte del equilibrio en las relaciones de poder entre el principal y el agente, lo que debe trascender no solo a nivel de discurso, sino también a nivel de práctica institucional en un contexto de rendición de cuentas, como un resultado en sí mismo y no sólo en el aspecto del proceso en las relaciones entre universidades y Estado.

\section{METODOLOGÍA}

Esta investigación de carácter cualitativo se encuentra circunscrita bajo los preceptos de la Teoría Fundamentada (Strauss y Corbin, 2002), donde la recolección de los datos, la selección de los casos y el análisis estuvieron estrechamente vinculados, siguiendo la lógica del método de la Teoría Fundamentada. En este sentido, los datos cualitativos fueron capturados mediante la realización de una serie de entrevistas semi estructuradas, elaborando una pauta con la selección de los temas y conceptos sensibilizadores, que hacen referencia a la implementación del financiamiento por desempeño en las universidades, los compromisos académicos e institucionales asumidos y el impacto en la autonomía de dichas instituciones.

Respecto a la validación de la entrevista, se utiliza la perspectiva de Kvale (2007), para quien la validación se vincula a la capacidad del investigador para cuestionar e interpretar teóricamente los hallazgos. Para la realización de las entrevistas semi-estructuradas se elaboró una pauta de entrevista, donde se definieron los siguientes temas sensibilizadores: situación de la universidad antes de la implementación del convenio de desempeño piloto, en aspectos como la docencia, investigación y gestión, la dinámica de la toma de decisiones asociadas al convenio y la relación universidad/ministerio de educación, los beneficios y 
dificultades percibidas con la implementación. El muestreo fue polietápico, seleccionando, en primera instancia, dos universidades pertenecientes al Consejo de Rectores de Universidades Chilenas (CRUCH), que compartían la característica de haber implementado al menos 5 proyectos de convenio de desempeño con el Ministerio de Educación durante el período 2007-2016, específicamente que estas universidades hubieran participado en la experiencia piloto de implementación de los convenios de desempeño en las universidades chilenas, según lo dispuesto en la Resolución Exenta de Educación № 0300, del 19 de julio de 2006.

Ambas instituciones tienen similar tamaño, están ubicadas en regiones territoriales y cuentan con una matrícula aproximada superior a los 8000 estudiantes. Ambas instituciones cuentan con una acreditación institucional vigente de 5 años, reconocimiento otorgado por la Comisión Nacional de Acreditación de Chile. Posteriormente, la selección de los sujetos a entrevistar estuvo determinada por el muestreo teórico, siguiendo la lógica propuesta por Strauss y Corbin (2002), que se diferencia del muestreo selectivo al estar guiado por la teoría emergente. El muestreo de la teoría fundamentada, por consiguiente, es secuencial, comenzando con un muestreo selectivo que pasa a ser un muestreo teórico cuando los conceptos comienzan a emerger, siendo el rol de los investigadores la definición de cuando pasar del muestreo teórico al muestreo selectivo (Draucker et al., 2007).

Desde esta perspectiva, el muestreo teórico se realizó secuencialmente el análisis de los datos, orientando las siguientes recolecciones, en orden de ir desarrollando los conceptos teóricos emergentes, siguiendo para este fin los lineamientos de Glaser (1978). En total, se entrevistaron 20 miembros de la comunidad académica, quienes desempeñaban diversas funciones al interior de las universidades, correspondiente a los perfiles de académicos, directivos y profesionales. Con la finalidad de complementar la información entregada por los miembros de las comunidades académicas de las universidades se entrevistaron a dos expertos, que tuvieron el rol de representar al Ministerio de Educación y ser los interlocutores con las universidades para la implementación de los convenios de desempeño pilotos, a quienes se les aplicó la misma pauta de entrevistas. Lo anterior además contribuye a disminuir el sesgo proveniente del conocimiento previo de los investigadores y que pudiera interferir en la interpretación de los datos, ya que según Strauss y Corbin (2002), obtener diferentes puntos de vista respecto a un acontecimiento permiten el distanciamiento del investigador. Cada uno de los participantes, antes de ser entrevistado, firmó un consentimiento informado, de acuerdo con lo establecido por los comités de ética nacionales, quedando claramente indicada la participación libre y voluntaria, manteniéndose además el anonimato y confidencialidad de la información recogida. Los entrevistados se referencian en género masculino, con la finalidad de salvaguardar la identidad de los participantes. Las entrevistas fueron realizadas durante el segundo semestre del año 2017. Asimismo, los resultados fueron presentados

La saturación teórica se determinó siguiendo los planteamientos de Glaser y Strauss (1967), quienes señalan que la saturación teórica se da en el instante en que no existen datos adicionales que puedan ser encontrados para el desarrollo de las categorías por parte de los investigadores. Realizada cada una de las entrevistas, se procedió a su transcripción de manera secuencial para generar el corpus textual, siendo procesadas en el software Nvivo 11, que facilita la aproximación a la teoría fundamentada (Hutchison et al., 2010). Los análisis se iniciaron, a través del método de comparación constante, con el descubrimiento de conceptos, mediante la codificación abierta, donde se comenzó a abrir el corpus textual; posteriormente, se dio paso a la identificación de las similitudes y diferencias, lo que permitió ir generando las categorías en términos de propiedades y dimensiones en nodos libres. Según Strauss y Corbin (2002) esta técnica permite pensar de manera comparativa al contrastar los incidentes con otros, lo cual permite la obtención de diversos puntos de vista, respecto a un hecho, lo que a su vez permite controlar el sesgo en la etapa del análisis.

\section{ANÁLISIS Y DISCUSIÓN DE RESULTADOS}

A continuación, los análisis se estructuran según los nodos identificados convenio de desempeño, rendición de cuentas en las universidades chilenas, y autonomía

\section{Convenio de Desempeño}

En primera instancia, destacan las percepciones respecto al desempeño en las universidades chilenas, como parte de una política de financiamiento, donde se destaca la noción de gestión del cambio y el impacto que tenía para la gestión institucional la inyección de recursos en el desarrollo y aceleración de procesos. "Los convenios de desempeño vinieron a acelerar procesos, revisar los procesos que estábamos haciendo, generar nuevos caminos de acción, nuevas iniciativas, fortalecer todo lo que decía relación con la capacitación de profesores, todos temas tremendamente relevantes" (E12). 


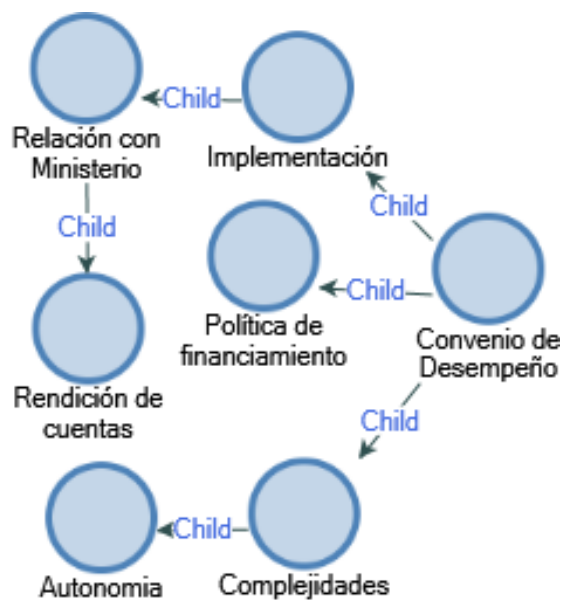

Fig. 1. Diagrama de análisis

El impacto que presenta para las universidades la implementación de esta forma de financiamiento se ve reflejado en el comentario siguiente: "el convenio como que cambio el paradigma y empezaron a sumarse los convenios, lo firmaba el rector y detrás del convenio de desempeño estaba toda una estructura de gobierno institucional, o sea, estaba el rector, el vicerrector, como uno de los gestores y responsables de la ejecución de los convenios de desempeño y toda la estructura posterior" (E6); al contar con el equipo central de la institución de educación superior, se asegura el cumplimiento de los compromisos adquiridos. "Los convenios de desempeño en su estructura misma entregaban una buena cantidad de recursos, a costa obviamente de lograr ciertas mejoras, introducían ciertos indicadores. Fue una visión nueva [de] como abordar los problemas, que fue bastante compleja, porque obviamente hubo que cambiar toda una cultura de cómo se desarrollaba todo lo que era la gestión universitaria y las responsabilidades que de alguna manera tenía que asumir cada una de las unidades, cada una de las personas que de alguna manera [se] involucraban" (E11).

Lo anterior coincide con el hecho de que los modelos de financiamiento basado en desempeño buscan precisamente incrementar la eficiencia y la productividad de la institución, lo que trae consigo un impacto en el cambio organizacional (Thornton y Friedel, 2016). El programa de convenios para el desempeño se inició su ejecución en diciembre del año 2007, para favorecer en las universidades el desarrollo de un plan de fortalecimiento que les permitiera alcanzar los más altos estándares de calidad. Por otra parte, pero en esta misma línea de financiamiento por desempeño, el convenio marco busca fortalecer las universidades estatales, a través del financiamiento necesario para que puedan cumplir su misión en el conjunto de las directrices de las políticas públicas. El convenio marco, por consiguiente, es un mecanismo de vinculación y financiamiento por desempeño del Ministerio de Educación con las universidades estatales, donde se otorga recursos públicos para temas específicos que conllevan a la generación de bienes públicos y al fortalecimiento de las instituciones y que incrementan su contribución al entorno (Palma y Rodríguez-Ponce, 2017)

\section{Rendición de cuentas en las universidades chilenas}

En Chile, el propósito general del instrumento convenio de desempeño es efectivamente lograr y favorecer la rendición de cuentas (Reich et al., 2011). En efecto, la rendición de cuentas en los convenios de desempeño se percibe diferente a la rendición de cuentas tradicional. Como dice un entrevistado: "el convenio es subscrito por el rector y el rector se compromete a determinados resultados, denominados entre comillas notables o sobresalientes, que en el lapso de tiempo que dure el convenio, 3 años, se rinden cuentas anualmente: Si la rendición de cuentas es insatisfactoria, el convenio faculta al Ministerio para, primero, congelar la entrega de recursos y, segundo, poder retirar el convenio y la institución debe de restituir los valores" (E4). Las universidades públicas son convocadas, en este contexto, a recolectar, reportar y analizar por medio de una serie de indicadores de desempeño su respuesta a las políticas de rendición de cuentas definidas por el Estado, entidad que, a su vez, incorpora con mayor frecuencia las demandas de información (Rutherford y Rabovsky, 2014) y que matiza claramente las consecuencias del incumplimiento del contrato.

Lo anterior tiene implicancias dentro de los sistemas de gobierno universitario, donde los marcos de regulación determinan fuertemente la capacidad de implementar de manera eficiente las medidas vinculadas a la calidad. Cuando las universidades no se benefician de una autonomía significativa, la implementación de acciones de eficiencia podría ser más difícil, sea dentro de la institución o a través de mecanismos de cooperación (Estermann et al., 2013). 


\section{Autonomía}

La implementación del financiamiento por desempeño resulta ser un caso de interés, si consideramos que la generación de la relación contractual más bien jerárquica que propone exige que cada unidad organizativa implicada en el proceso de cuenta de su desempeño y, producto de ello, la rendición de cuentas y la eficiencia deban aumentarse (Barnes et al., 2014). Teniendo en cuenta además el hecho de que la autonomía de las universidades y su control han cambiado en los últimos años (Nagy et al., 2014).

"[Hubo] un fuerte interés [de parte] del Ministerio para que efectivamente esto quedara instalado [Convenio de Desempeño] y efectivamente fuera un tema positivo para las universidades y después utilizarlo como mecanismo de financiamiento, de ahí para delante, un tema un tanto perverso que obviamente el que pone la plata pone la música, como se dice, por lo cual, las orientaciones que nos daba el ministerio eran muy estrictas y que estas se acentuaron posteriormente, por ejemplo, en los convenios de desempeño de armonización curricular, donde nos fijaron ciertas condiciones que había que cumplir, digamos, y eso a veces, tensionaba mucho el trabajo interno" (E11). Por consiguiente, la autonomía se configura como parte de las complejidades que trae consigo la implementación de los convenios de desempeño en las universidades.

De acuerdo con lo anterior, las interacciones que ocurren en procesos de autonomía institucional se producen en un contexto de derechos acordados y regulados, donde los mecanismos definidos tienen el rol de ayudar a determinar el apropiado balance entre la autonomía y la rendición de cuentas, situación que refleja el reconocimiento de la existencia de un interés público y la necesidad de reconciliar la educación terciaria con los beneficios de la autonomía institucional (Deb, 2016). En este sentido, respecto a la autonomía, se reconoce que "La universidad tenía autonomía dentro del margen reglamentario que fijó el Ministerio, o sea, nosotros ejecutábamos libremente los gastos, los rendíamos. Si había cosas que no correspondían de acuerdo con los instructivos del ministerio se nos hacía ver, hacíamos las reformulaciones, pero dentro del marco reglamentario de ejecución de los recursos" (E12). Esta percepción coincide con la noción de autonomía, donde ésta se encuentra intrínsecamente ligada a un marco regulatorio y contextual, dentro del cual la institución puede operar. Cabe recordar que uno de los aspectos cruciales para la gobernanza en el sistema de educación superior corresponde al reconocimiento de la autonomía de las instituciones y su responsabilidad (Brunner y Ganga, 2016), resultando muchas veces contradictorio que determinadas políticas de financiamiento basadas en el desempeño afecten o reduzcan la autonomía de las instituciones de educación superior.

La forma de financiamiento descrita trae consigo cambios que podrían ser estructurales, programáticos o procedimentales y eso podría afectar a la práctica institucional o a la propia política (Thornton y Friedel, 2016). Resulta evidente, por tanto, que la relación contractual que surge en el financiamiento por desempeño entre el gobierno y las instituciones de educación superior va más allá del simple cumplimiento de los resultados comprometidos, puesto que afecta los procesos en una lógica de monitoreo y control permanente que genera cambios en el proceso mismo y no sólo en los resultados. De igual manera, vale precisar que, a primera vista, los términos de autonomía y rendición de cuentas no parecieran presentar problemas. En términos simples, autonomía significa, en sentido amplio, poder gobernar sin casi controles externos, mientras que la rendición de cuentas implica el requisito de demostrar acciones responsables a algún externo. Aunque en teoría se ha argumentado que no hay incompatibilidad necesaria entre ser altamente autónomo y rigurosamente responsable, en la práctica, se siente que normalmente se requiere más responsabilidad y menos autonomía. Lo ideal es generar un equilibrio entre ambas condiciones, ya que demasiada autonomía podría llevar a que las universidades no respondan a la sociedad y, por otra parte, demasiada responsabilidad puede destruir el ethos académico necesario (Berdahl, 1990).

No obstante, es posible encontrar opiniones divergentes al respecto, donde la rendición de cuentas "tiene resistencia interna desde quienes tienen que gestionar el convenio, resistencia a sentirse controlado periódicamente o resistencia a tener que rendir cuentas periódicamente respecto de su quehacer: Respecto al control al poco andar de tiempo, a la mitad del convenio, el equipo del mismo comienza a darse cuenta que esa persona (negociador) le agrega valor al convenio, nos muestra otras miradas que nosotros no estábamos teniendo y en vez de ser una persona que controla, se transforma en un facilitador para nosotros, yo diría...eso" (E4). En este marco de actuación, se podría esperar una cierta hostilidad hacia la rendición de cuentas si consideramos que las instituciones de educación superior han disfrutado tradicionalmente de una considerable deferencia y autonomía; sin embargo, las respuestas recibidas en un estudio realizado por Hicklin et al (2012) son bastante diversas, ya que una minoría de los rectores entrevistados expresa una respuesta escéptica a estos esfuerzos. Por otra parte, la gran mayoría se mostraba más optimista, sugiriendo que los convenios de desempeño eran apropiados para que los representantes públicos avanzaran en sus objetivos a través de este tipo de políticas, manteniendo la diversidad de puntos de vista sobre la legitimidad de las políticas implementadas incluyendo las de financiamiento. 
Resulta útil señalar que esta figura del negociador, que tuvo inicialmente la implementación de los convenios de desempeño en Chile, "resguardaba los intereses del Ministerio y se producía un gallito, pero con altura de miras, donde primaba el criterio académico, considerando las capacidades de la Institución y las necesidades que el Ministerio" (E1). En efecto, el negociador era un experto designado por el Ministerio de Educación para liderar el proceso de negociación del Plan de Mejoramiento Institucional inicial, definido en el marco del convenio de desempeño, y concretar así los desempeños notables, clave para la definición de metas ambiciosas, siendo responsables además del monitoreo y seguimiento del convenio a lo largo de su implementación (Reich et al., 2011). Efectivamente, se reconoce este rol, en el sentido de que "fue muy bueno para el proyecto, la visita de los negociadores, porque en el fondo quitan la barrera de auto-referencialidad que tú puedas llegar a tener, con la fijación de indicadores muy autoflagelantes, muy exigentes o indicadores muy autocomplacientes, o sea, esa cuestión la veía muy bien el negociador y eso también yo creo que fue muy potente" (E15). Por consiguiente, puede asignarse a la figura del negociador un rol clave para el logro de un equilibrio entre los intereses del principal (gobierno) y el agente (universidad), siendo necesario para la percepción de la autonomía el considerar dentro de la discusión las capacidades y perspectivas institucionales de la universidad, particularmente los compromisos de desempeño académicos a asumir por parte de ésta. De igual forma, se percibe cierta resistencia al momento de implementar los convenios de desempeño por parte de miembros de la comunidad académica quienes se preguntaban "por qué tenía que venir alguien de afuera a decir lo que teníamos que hacer, se pierde la autonomía universitaria, se pasa por encima de los decanos. Estaba un poco ese resquemor" (E6)., percibiéndose cierta pérdida de autonomía en lo que respecta a la toma de decisiones referidas a los gastos. Con orientaciones estrictas respecto a la agenda que se debía cumplir, lo que genera cierta tensión al interior de las instituciones de educación superior. Lo anterior coincide con una mayor carga de regulaciones que durante los últimos treinta años se ha experimentado en los países que poseen una mayor capacidad de gestión de políticas públicas, desde los gobiernos hacia las universidades, redefiniéndose de esta forma la noción de autonomía al interior de las universidades (Bernasconi, 2015).

De esta forma, la autonomía universitaria se contextualiza y manifiesta en el día a día de la ejecución del proyecto, donde el control y seguimiento van mermando derechamente la autonomía universitaria, en la capacidad de decisión respecto a aspectos tales como modificar la naturaleza de un gasto, con miras a la consecución de los resultados, o la optimización de los recursos: "Hoy día, se transforma nuevamente en que si tú quieres, cambiar una aguja por un alfiler, tienes que hacer nuevamente la presentación al DFI, al Departamento de Financiamiento Institucional en Santiago, que te entreguen a ti, la no objeción o el visto bueno en el cambio y ahí recién tú puedes ejecutar" (E4). Lo anterior refleja una situación de complejidad para las universidades, debido a que lograr un punto de equilibrio entre los requerimientos y el control de parte del Ministerio y las necesidades y dificultades que manifiestan en su día a día las universidades en la ejecución de los convenios de desempeño genera efectos sobre la autonomía universitaria puesto que en muchas ocasiones las exigencias centradas en lo operacional van en contra de lo especificado en el plan de desarrollo de la institución y en algunos casos inclusive se llega a presentar la paradoja de que por realizar una actividad vinculada al convenio se duplican esfuerzos y o gastos predefinidos en la institución a partir del plan determinado con antelación a la existencia del convenio.

Más aún si se toma en consideración que las prioridades del principal y del agente podrían diferir, causando problemas debido a la separación de intereses (Enders et al., 2013). Las elecciones estratégicas de la universidad podrían no coincidir con las del gobierno, traduciéndose esto en problemas de agencia (Magalhães et al., 2013). Debemos tener en cuenta, por lo tanto, que la relación contractual de los convenios de desempeño es más bien jerárquica, mientras que las instituciones universitarias utilizan su autonomía legal en la declaración de su misión, la definición de sus estatutos, la gestión de sus recursos y la realización actividades. Las discrepancias pueden ser un buen elemento de reflexión, además de facilitar los mecanismos de autorregulación para la dirección política de las instituciones públicas autónomas y el debate sobre la gobernanza universitaria. Aunque la rendición de cuentas puede ser considerada un obstáculo, particularmente para el equipo que lidera la institución de educación superior, por las decisiones cada vez más complejas que les obliga a tomar (Pucciarelli y Kaplan, 2016), un escenario de financiamiento contra resultados implica necesariamente el replanteamiento de la relación con las universidades, al evidenciar la necesidad de que estas sean más rentables y eficientes en la utilización de los recursos (Araneda-Guirriman y Pedraja-Rejas, 2016). Más aún si se considera lo planteado por Morales (2014) quien establece que, en el caso de Chile, la reforma que incorporó mecanismos de evaluación por desempeño no generó los resultados esperados desde el diseño teórico.

Si creemos que la universidad requiere de autonomía respecto a una política o influencia corporativa para operar de manera adecuada, el Estado como guardián de la universidad en aspectos relevantes deberían de garantizar el financiamiento fiscal y, al menos en Europa continental, un fuerte autogobierno profesional y protección de la libertad académica (Enders et al., 2013). En el caso del sistema de educación superior chileno, la implicación clara del gobierno nacional se hace difícil por carecer de un lineamiento claro sobre el desarrollo del sistema en aspectos como la expansión de la matrícula, es decir, qué áreas, qué combinación de educación presencial y virtual, qué monto se debiera aumentar del gasto público y el manejo del financiamiento público (Brunner y Ganga, 2016). 


\section{CONCLUSIONES}

El financiamiento por desempeño es un mecanismo implementado con la finalidad de optimizar la utilización de los recursos provenientes del Estado, promoviendo de esta forma la eficiencia y transparencia en las instituciones de educación superior y vinculando los recursos entregados al logro de resultados concretos previamente acordados con el Estado; es decir, no son de libre elección por parte de las universidades. Si bien es cierto que la naturaleza de este mecanismo es facilitar el cumplimiento de la misión y el proyecto estratégico que definen estas instituciones, la realidad es que nos obliga a preguntarnos por la autonomía al contemplar un componente direccionado por el gobierno. De acuerdo con miembros de las comunidades académicas entrevistados, se percibe que la implementación de los convenios de desempeño se encuentra estrechamente vinculada con la rendición de cuentas públicas, lo que inevitablemente genera una situación de control y monitoreo permanente tanto al interior de la universidad como por parte del Estado. Lo anterior, se configura, por tanto, en una situación de complejidad, puesto que los líderes de estas instituciones se ven obligados a rendir cuentas de forma constante de los recursos públicos recibidos, al mismo tiempo que tienen que vencer resistencias internas al incorporar la rendición un monitoreo y control de logros con énfasis en los procesos administrativos, financieros y académicos. Se vuelve necesario, en este contexto, el contar con un equipo de profesionales que se ocupen de todos los procesos y procedimientos administrativos asociados a la ejecución del gasto y a la posterior rendición de cuentas.

Por consiguiente, estos procesos vinculados a la rendición de cuentas cuestionan también lo que ocurre finalmente con la autonomía de dicha institución, si consideramos que un mayor control sobre el gasto de los recursos limita la capacidad de decisión y la definición del qué, cómo y cuándo gastar los recursos para cumplir con los objetivos y resultados comprometidos. A pesar de ello, la inyección de recursos públicos que reciben las universidades por la vía del convenio de desempeño permite la elección de inversiones estratégicas y acelerar procesos que estén en la línea del cumplimiento de su plan de desarrollo institucional. Quizás, la cuestión es lograr un punto de equilibrio entre ayudas y requerimientos externos e intereses internos y poner énfasis en el cumplimiento de los resultados, generando espacios de confianza respecto al control y monitoreo de los procesos. Si bien se toma en cuenta la literatura y las investigaciones revisadas en el contexto nacional que abordan la autonomía al interior de las universidades, este análisis se efectúa desde una perspectiva más amplia desde la gobernanza, la teoría de agencia y la política pública, por mencionar algunas visiones, pero no desde una mirada cualitativa que aborde y rescate la percepción que tienen los propios actores de este concepto, que puede resultar abstracto, en el contexto de la implementación de un mecanismo de financiamiento por desempeño. Contribuyendo de esta forma con una aproximación de los efectos secundarios que son identificados por los actores, como mayor restricción, control, limitaciones a la autonomía universitaria en la utilización y decisión en torno a los recursos recibidos.

Finalmente, de cara a investigaciones futuras se hace preciso investigar más sobre la naturaleza, sentido y efectos de los convenios de desempeño, desarrollando modelos explicativos ('modelos de') y para la intervención ('modelos para'). $Y$ en este contexto, y al contemplar un componente direccionado por el gobierno, se puede establecer la necesidad de generar un modelo multinivel que sea capaz de evaluar los efectos directos e indirectos de este mecanismo de financiamiento en las instituciones de educación superior.

\section{AGRADECIMIENTOS}

Los autores agradecen el patrocinio de la Comisión Nacional de Investigación Científica y Tecnológica de Chile, CONICYT a través del proyecto FONDECYT Regular № 1171276 y del proyecto UTA Mayor 8741-17.

\section{REFERENCIAS}

Araneda-Guirriman, C. A. y L. M. Pedraja-Rejas, Financiamiento por Desempeño en Chile: Análisis Conceptual de un Instrumento para la Educación Superior, Formación Universitaria, 9(3), 75-86 (2016)

Barnes, A., G. Brown y S. Harman, Global Politics of Health Reform in Africa: Performance, Participation and Policy, Springer (2014)

Berdahl, R., Academic Freedom, Autonomy and Accountability in British Universities, doi: https://doi.org/10.1080/03075079012331377491, Studies in Higher Education, 15(2), 169-180 (1990)

Bernasconi, A., El gobierno de las instituciones. La educación superior de Chile: Transformación, desarrollo y crisis, 259293 (2015)

Brunner, J.J. y F.A. Ganga, Gobernanza de la educación superior: una mirada crítica y propositiva al caso de Chile, Revista Dilemas Contemporáneos: Educación, Política y Valores, 1(33), 1-25 (2016)

Christopherson, S., M. Gertler y M. Gray, Universities in Crisis, doi:10.1093/cjres/rsu006, Cambridge Journal of Regions, Economy and Society 7, 209-215 (2014)

Correa, J., Autonomía de las Instituciones de Educación Superior en el Proyecto de Ley de Educación Superior. Una mirada desde la Perspectiva de los Criterios de la Comunidad Europea, Centro de Estudios Públicos, 98 p. (2017) 
Deb, B., Governance in Higher Education in the Context of Assam, doi: 10.15373/2249555X, Indian Journal of Applied Research, 6(2) (2016)

Dougherty, K. J., S. M. Jones y otros cuatro autores, Looking Inside the Black Box of Performance Funding for Higher Education: Policy Instruments, Organizational Obstacles, and Intended and Unintended Impacts; doi: 10.7758/rsf.2016.2.1.07, RSF: The Russell Sage Foundation Journal of the Social Sciences, 2(1), 147-173 (2016)

Dougherty, K.J., y V. Reddy, Performance Funding for Higher Education: What Are the Mechanisms What Are the Impacts, doi: 10.1002/aehe.20008, ASHE Higher Education Report, 39 (2) John Wiley y Sons (2013)

Draucker, C.B., D.S. Martsolf, R. Ross y T. B. Rusk, Theoretical Sampling and Category Development in Grounded Theory. Qualitative Health Research, 17(8), 1137-1148 (2007)

Enders, J., H. De Boer y E. Weyer, Regulatory Autonomy and Performance: The Reform of Higher Education revisited, doi: https://doi.org/10.1007/s10734-012-9578-4, Higher Education, 65(1), 5-23 (2013)

Estermann, T., E.B. Pruvot y A. L. Claeys-Kulik, Designing Strategies for Efficient Funding of Higher Education in Europe, DEFINE interim report, Brussels (2013)

European University Association, Declaración de Graz Después de Berlín: el papel de las universidades (2003)

Glaser, B. G., Theoretical sensitivity: Advances in the Methodology of Grounded Theory, Sociology Pr. (1978)

Hicklin, A., T. Rabovsky y D.P. Moynihan, Performance Funding in Higher Education: Exploring Perceptions of Accountability Policies, In APSA 2012 Annual Meeting Paper (2012)

Hutchison, A. J., L. H. Johnston y J. D. Breckon, Using QSR-NVivo to Facilitate the Development of a Grounded Theory Project: an Account of a Worked Example; doi: https://doi.org/10.1080/13645570902996301, International Journal of Social Research Methodology, 13(4), 283-302 (2010)

Kairuz, T., L. Andriés, T. Nickloes e I. Truter, Consequences of KPIs and Performance Management in Higher Education, doi: https://doi.org/10.1108/IJEM-05-2015-0067, Inter. Journal of Educational Management, 30(6), 881-893 (2016)

Kivistö, J., e I. Zalyevska, Agency theory as a framework for higher education governance. In The palgrave international handbook of higher education policy and governance, Palgrave Macmillan, London, 132-151 (2015)

Kvale, S., Doing Interviews, Conducting an Interview, Methods, 34-51 (2007)

Letizia, A., Performance-Based Funding in Higher Education: The State of Truth in the Information Age, Lexington Books (2016)

Maassen, P., A New Social Contract for Higher Education? In Higher education in societies, doi: https://doi.org/10.1007/978-94-6209-746-9_4, SensePublishers, 33-50 (2014)

Magalhães, A., A. Veiga, A. Amaral, S. Sousa y F. Ribeiro, Governance of Governance in Higher Education: Practices and lessons drawn from the Portuguese case, doi:10.1111/hequ.12021, Higher Education Quarterly, 67(3), 295-311 (2013)

Marginson, S.W., Global Trends in Higher Education Financing: The United Kingdom; doi: https://doi.org/10.1016/j.ijedudev.2017.03.008, International Journal of Educational Development (2017)

Morales, M., Nueva Gestión Pública en Chile, Revista de Ciencia Política (Santiago), 34(2), 417-438 (2014)

Nagy, S.G., G. Kováts y A.O. Németh, Governance and Funding of Higher Education-International Trends and Best Practices; doi: https://doi.org/10.1016/j.sbspro.2014.01.190, Procedia-Social and Behavioral Sciences, 116, 180-184 (2014)

Palma-Quiroz, A. y E. Rodríguez-Ponce, La asignación de recursos a las universidades del Estado en un contexto de rendición de cuenta: la lógica de los Convenios Marco en el Caso de Chile, Ingeniare. Rev. Chilena de Ing., 25(2), $192-195$ (2017)

Pliscoff-Varas, C., Implementando la nueva gestión pública: problemas y desafíos a la ética pública, El caso chileno, Convergencia, 24(73), 141-164 (2017)

Pucciarelli, F. y A. Kaplan, Competition and Strategy in Higher Education: Managing Complexity and Uncertainty; doi: https://doi.org/10.1016/j.bushor.2016.01.003, Business Horizons, 59(3), 311-320 (2016)

Reich Albertz, R., F. Machuca y otros cinco autores, Bases y Desafíos de la Aplicación de Convenios de Desempeño en la Educación Superior de Chile, Ingeniare. Revista Chilena de Ingeniería, 19(1), 08-18 (2011)

Rutherford, A. y T. Rabovsky, Evaluating Impacts of Performance Funding Policies on Student Outcomes in Higher Education; doi: https://doi.org/10.1177/0002716214541048, The ANNALS of the American Academy of Political and Social Science, 655(1), 185-208 (2014)

Strauss, A. L. y J. Corbin, Bases de la Investigación Cualitativa: Técnicas y Procedimientos para Desarrollar la Teoría Fundamentada, Medellín, Universidad de Antioquia (2002)

Thornton, Z. M. y J. N. Friedel, Performance-based Funding: State Policy Influences on Small Rural Community Colleges; doi: https://doi.org/10.1080/10668926.2015.1112321, Community College J. of Research and Practice, 40(3), 188-203 (2016)

Trow, M., Trust, Markets and Accountability in Higher Education: A Comparative Perspective, doi: https://doi.org/10.1016/S0952-8733(96)00029-3, Higher Education Policy, 9(4), 309-324 (1996)

Yutronic, J., R. Reich Albertz, y otros tres autores, Convenios de Desempeño y su Aporte en el Financiamiento de la Educación Superior en Chile, Educación Superior y Sociedad, 16(1), 53-77 (2012) 